\title{
THE STRUCTURE OF CERTAIN UNITARY REPRESENTATIONS OF INFINITE SYMMETRIC GROUPS
}

\author{
BY \\ ARTHUR LIEBERMAN
}

\begin{abstract}
Let $S$ be an infinite set, $\beta$ an infinite cardinal number, and $G_{\beta}(S)$ the group of those permutations of $S$ whose support has cardinal number less than $\beta$. If $T$ is any nonempty set, $S^{T}$ is the set of functions from $T$ to $S$. The canonical representation $\Lambda_{\beta}^{T}$ of $G_{\beta}(S)$ on $L^{2}\left(S^{T}\right)$ is the direct sum of factor representations. Factor representations of types $\mathrm{I}_{\infty}, \mathrm{II}_{1}$, and $\mathrm{II}_{\infty}$ occur in this decomposition, depending upon $S, \beta$, and $T$; the type $\mathrm{II}_{1}$ factor representations are quasi-equivalent to the left regular representation.

Let $G_{\beta}(S)$ have the topology of pointwise convergence on $S . G_{\beta}(S)$ is a topological group but is not locally compact. Every continuous representation of $G_{\beta}(S)$ is the direct sum of irreducible representations. Let $\Gamma$ be a nontrivial continuous irreducible representation of $G_{\beta}(S)$. Then $\Gamma$ is continuous iff $\Gamma$ is equivalent to a subrepresentation of $\Lambda_{\beta}^{T}$ for some nonempty finite set $T$ iff there is a nonempty finite subset $Z$ of $S$ such that the restriction of $\Gamma$ to the subgroup of those permutations which leave $Z$ pointwise fixed contains the trivial representation of this subgroup.
\end{abstract}

I. Introduction. The representations of the symmetric group on a finite set have been studied in great detail. Little has been done toward analyzing and classifying the representations of the symmetric groups on an infinite set. Thoma [7] has found all type $\mathrm{II}_{1}$ factor representations of the group of finite permutations of a countably infinite set; he did this by explicitly constructing the characters of the group.

If $S$ is an infinite set and $\beta$ is a cardinal number, let $G_{\beta}(S)$ be the group of all permutations $p$ of $S$ such that the cardinal number of $\{s \in S: p(s) \neq s\}$ is less than $\beta$.

Below we analyze the canonical representation $\Lambda_{\beta}^{T}$ of $G_{\beta}(S)$ on $L^{2}\left(S^{T}\right)$, where $T$ is any nonempty set and $S^{T}$ is the set of functions with domain $T$ and range contained in $S . \Lambda_{\beta}^{T}$ is the direct sum of factor representations of $G_{\beta}(S)$. The factor representations in the decomposition are of types $\mathrm{I}_{\infty}, \mathrm{II}_{1}$, and $\mathrm{II}_{\infty}$, depending upon $S, T$, and $\beta$; the type $\mathrm{II}_{1}$ factor representations are all quasi-equivalent to the left regular representation of $G_{\beta}(S)$.

Assume now that $T$ is finite and nonempty. Then the von Neumann algebra generated by $\Lambda_{\beta}^{T}\left(G_{\beta}(S)\right)$ is the same for all infinite cardinal numbers $\beta . \Lambda_{\beta}^{T}$ is the direct sum of finitely many infinite-dimensional irreducible representations of $G_{\beta}(S)$. The set of equivalence classes of irreducible subrepresentations of the

Presented to the Society, September 2, 1971; received by the editors August 31, 1970 and, in revised form, March 5, 1971.

AMS 1970 subject classifications. Primary 22A25; Secondary 20E99, 22D10.

Key words and phrases. Infinite symmetric groups, representations of not locally-compact groups, representations of discrete groups.

Copyright (C) 1972, American Mathematical Society 
representation $\Lambda_{\beta}^{T}$ of $G_{\beta}(S)$, as the cardinal number of $T$ varies over the positive integers, is in a canonical one-to-one correspondence with the set of equivalence classes of irreducible representations of the symmetric group on $n$ symbols, as $n$ ranges over the positive integers.

Let $G_{\beta}(S)$ have the topology of pointwise convergence on $S . G_{\beta}(S)$ is a topological group but is not locally compact. We will say that a representation of $G_{\beta}(S)$ is continuous if it is continuous with respect to this topology.

$\Lambda_{\beta}^{T}$ is continuous if $T$ is finite. We show that any continuous representation of $G_{\beta}(S)$ is the direct sum of irreducible representations. Any nontrivial continuous irreducible representation of $G_{\beta}(S)$ is equivalent to a subrepresentation of $\Lambda_{\beta}^{T}$ for some nonempty finite set $T$.

We further characterize the continuous irreducible representations of $G_{\beta}(S)$ by showing that the following are equivalent if $\Gamma$ is an irreducible representation of $G_{\beta}(S)$ :

1. $\Gamma$ is continuous.

2. $\exists S_{1} \subseteq S$ such that $S_{1}$ is finite and the restriction of $\Gamma$ to the subgroup of $G_{\beta}(S)$ of those permutations which leave $S_{1}$ pointwise invariant contains the trivial representation of this subgroup.

3. There is a nonnegative integer $n$ such that condition 2 holds for any subset $S_{2}$ of $S$ if $S_{2}$ has cardinal number $\geqq n$.

The results in this paper are contained in the author's doctoral thesis, which was written at the Massachusetts Institute of Technology under the supervision of Professor I. E. Segal. The author wishes to thank Professor Segal for suggesting this topic and for supervising the thesis. This research was supported by a National Science Foundation Graduate Fellowship.

II. Lemmas and notation. The following lemmas will be needed. Lemma 3 is well known; we include it for completeness.

Lemma 1. Let $G$ be a group. Let $H_{i}, i=1,2$, be a Hilbert space. Let $\Gamma_{i}, i=1,2$, be a representation of $G$ on $H_{i}$. Let $Z \subseteq G$. Assume

1. $\Gamma_{1} \sim \Gamma_{2}$.

2. $\exists v \in H_{1}, v \neq 0$, such that $\Gamma(g) v=v$ if $g \in Z$.

Then $\exists w \in H_{2}, w \neq 0$, such that $\Gamma_{2}(g) w=w$ if $g \in Z$.

Proof. Since $\Gamma_{1} \sim \Gamma_{2}$, there exist cardinal numbers $\alpha_{1}, \alpha_{2}$ such that $\alpha_{1} \Gamma_{1} \cong \alpha_{2} \Gamma_{2}$. $\alpha_{i} \Gamma_{i}$ acts on $\bigoplus_{a \in \alpha_{i}} H_{i a}$, where $H_{i a}=H_{i}$ for all $a \in \alpha_{i}$. Let $V$ be a unitary operator which implements the equivalence between $\alpha_{1} \Gamma_{1}$ and $\alpha_{2} \Gamma_{2}$. If $b \in \alpha_{i}$, for $i=1,2$, let $P_{i b}$ be projection onto the subspace $H_{i b}$ of $\bigoplus_{a \in \alpha_{i}} H_{i a}$.

Let $v \in H_{1}$ be chosen as in hypothesis 2 . Let $w$ in $\bigoplus_{a \in \alpha_{i}} H_{i a}$ be chosen so that $P_{10} w=v$ and $P_{1 a} w=0$ if $a \in \alpha_{1}$ and $a \neq 0$. Note that $\bigoplus_{a \in \alpha_{2}} P_{2 a}$ is the identity operator on $\bigoplus_{a \in \alpha_{2}} H_{2 a}$. Therefore there is an element $c$ in $\alpha_{2}$ such that $P_{2 c} V w \neq 0$. Let $x=P_{2 c} V w$. Let $g \in Z$. Then $\Gamma_{2}(g) x=\Gamma_{2}(g) P_{2 c} V w=P_{2 c} \Gamma_{2}(g) V w=P_{2 c} V \Gamma_{1}(g) w$ $=P_{2 c} V w=x$. 
Lemma 2. Let $G$ be a group, $H$ a Hilbert space, and $\Gamma$ a representation of $G$ on $H$. Let $J$ be a closed subspace of $H, P$ be projection onto $J$, and $G_{J}=\{g \in G: \Gamma(g) J=J\}$. Assume

a. $\exists X \subseteq G: H=\bigoplus_{x \in X} \Gamma(x) J$.

b. If $g \in G$, then $\Gamma(g) J=J$ or $\Gamma(g) J \perp J$.

c. $P \in \Gamma(G)^{\prime \prime}$.

Then $T \rightarrow T P$ is an algebraic *-isomorphism from $\Gamma(G)^{\prime}$ onto $\Gamma(G)^{\prime} \mid J$.

$$
\Gamma(G)^{\prime}\left|J=\Gamma\left(G_{J}\right)^{\prime}\right| J .
$$

Proof. $T \rightarrow T P$ is an algebraic *-homomorphism from $\Gamma(G)^{\prime}$ onto $\Gamma(G)^{\prime} \mid J$. $T=\bigoplus_{x \in X} \Gamma(x) T P \Gamma\left(x^{-1}\right) \mid \Gamma(x) J$. Therefore $T=0$ if $T P=0$, so that $T \rightarrow T P$ is oneto-one.

If $g \in G-G_{J}$, then $P \Gamma(g) P=0$. Therefore $\Gamma(G)^{\prime \prime}\left|J=\Gamma\left(G_{J}\right)^{\prime \prime}\right| J$.

Lemma 3. Let $H$ be a Hilbert space, $G$ a group, and $\Gamma$ a representation of $G$ on $H$. Let $Q$ be a subset of $G, J=\{v \in H: \Gamma(g) v=v$ if $g \in Q\}$, and $P$ be projection onto $J$. Then $P \in \Gamma(G)^{\prime \prime}$.

Proof. Let $v \in J$ and $T \in \Gamma(G)^{\prime}$. Let $g$ be an arbitrary member of $Q$. Then $\Gamma(g) T v=T \Gamma(g) v=T v$. Therefore $T v \in J$. The conclusion follows immediately from the double commutant theorem.

Notation. Let $S$ be an arbitrary infinite set. $S$ will remain fixed for the remainder of this paper. If $\beta$ is an infinite cardinal number, $G_{\beta}$ is the set of permutations $p$ of $S$ such that the cardinal number of $\{s \in S: p(s) \neq s\}$ is less than $\beta$. Note that $G_{\beta}$ is the group of all permutations of $S$ if $\beta$ is sufficiently large.

Let $T$ be any set. Then $|T|$ will denote the cardinal number of $T . L^{2}(T)$ is the Hilbert space of all complex valued functions $f$ with domain $T$ such that $\|f\|<\infty$, where $\|f\|^{2}=\sum_{t \in T}|f(t)|^{2}$.

Let $Z$ be any set. $T^{Z}$ will denote the set of functions with domain $Z$ and range contained in $T . T^{Z i}=\left\{f \in T^{Z}: f\right.$ is $\left.1-1\right\} . T^{z b}=\left\{f \in T^{Z}: f\right.$ is $1-1$ and onto $\}$.

A partition of $T$ is a collection of nonempty disjoint subsets of $T$ whose union is $T . \mathscr{P}(T)$ will denote the set of partitions of $T$.

$G(T)$ denotes the group of all permutations of $T$. If $\beta$ is an infinite cardinal number, $G_{\beta}(T)$ denotes $\{\pi \in G(T):|\{t \in T: \pi(t) \neq t\}|<\beta\}$.

Recall [3, pp. 21-31] that a cardinal number $\alpha$ is also an ordinal number, and that $\alpha=\{$ ordinal numbers $\gamma: 0 \leqq \gamma<\alpha\}$.

III. The representation of $G_{\beta}$ on $L^{2}\left(S^{\alpha}\right)$. Let $\alpha$ be a nonzero cardinal number and $\beta$ be an infinite cardinal number. Let $\Omega_{\beta}^{\alpha}$ be the representation of $G_{\beta}$ on $L^{2}\left(S^{\alpha}\right)$ defined by $\Omega_{\beta}^{\alpha}(g) v(f)=v\left(g^{-1} f\right)$, for $g \in G_{\beta}, v \in L^{2}\left(S^{\alpha}\right)$, and $f \in S^{\alpha}$. If $g \in G_{\beta}$, let $\Lambda_{\beta}^{\alpha}(g)$ be the restriction of $\Omega_{\beta}^{\alpha}(g)$ to $L^{2}\left(S^{\alpha i}\right) ; \Lambda_{\beta}^{\alpha}$ is a representation of $G_{\beta}$.

The following theorem reduces the study of the representations $\Omega_{\beta}^{\alpha}$ of $G_{\beta}$ to that of the representations $\Lambda_{\beta}^{\alpha}$ of $G_{\beta}$. 
THEOREM 1. Let $\alpha$ and $\beta$ be cardinal numbers such that $\alpha>0$ and $\beta \geqq \aleph_{0}$. Then $\Omega_{\beta}^{\alpha} \cong \bigoplus_{\mathscr{P} \in \mathscr{P}_{(\alpha)}} \Lambda_{\beta}^{|\mathscr{P}|} \cong \bigoplus_{1 \leqq \gamma \leqq|S|} \zeta(\gamma) \Lambda_{\beta}^{\gamma}$, where $\zeta(\gamma)=|\{\mathscr{P} \in \mathscr{P}(\alpha):|\mathscr{P}|=\gamma\}|$ if $\gamma$ is a cardinal number and $1 \leqq \gamma \leqq|S|$.

Proof. The equivalence of $\bigoplus_{\mathscr{P}_{\in} \mathscr{P}_{(\alpha)}} \Lambda_{\beta}^{|\mathscr{P}|}$ with $\bigoplus_{1 \leqq \gamma \leqq|S|} \zeta(\gamma) \Lambda_{\beta}^{\alpha}$ is obvious.

If $f \in S^{\alpha}$, let $\mathscr{P}(f)=\left\{f^{-1}(s): s\right.$ is in the range of $\left.f\right\} . \mathscr{P}(f) \in \mathscr{P}(\alpha)$. Let $\theta(f)$ be the unique element of $S^{\mathscr{P}(f) i}$ such that $\theta f^{-1}(s)=s$ for all $s$ in the range of $f . \theta$ is a oneto-one mapping from $S^{\alpha}$ onto $\bigcup_{\mathscr{P}_{\in} \mathscr{P}_{(\alpha)}} S^{\mathscr{P} i}$.

Let $U$ be the mapping from $L^{2}\left(S^{\alpha}\right)$ to $\bigoplus_{\mathscr{P}_{\in} \mathscr{P}_{(\alpha)}} L^{2}\left(S^{\mathscr{P} i}\right)$ defined by $U v(h)=v\left(\theta^{-1} h\right)$ for $v \in L^{2}\left(S^{\alpha}\right)$ and $h \in \bigcup_{\mathscr{P}_{\in} \mathscr{P}_{(\alpha)}} S^{\mathscr{P}_{i}}$. $U$ is unitary and implements an equivalence

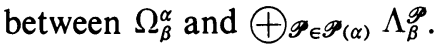

Notation. If $T \subseteq S$, let $P_{T}^{\alpha}$ be projection onto the subspace $L^{2}\left(T^{\alpha b}\right)$ of $L^{2}\left(S^{\alpha i}\right)$.

If $0<\alpha \leqq|S|$, define a representation $\Phi$ of $G(\alpha)$ on $L^{2}\left(S^{\alpha i}\right)$ by $\Phi(\pi) v(f)=v(f \pi)$, for $\pi \in G(\alpha), v \in L^{2}\left(S^{\alpha i}\right)$, and $f \in S^{\alpha i}$.

LEMMA 4. Let $\alpha$ and $\beta$ be cardinal numbers such that $\alpha>0$ and $\beta \geqq \boldsymbol{\aleph}_{0}$. Let $T \subseteq S$. Assume $|T|=\alpha$ and $|S-T| \geqq \aleph_{0}$. Then $P_{T}^{\alpha} \in \Lambda_{\beta}^{\alpha}\left(G_{\beta}\right)^{\prime \prime}$.

Proof. $P_{T}^{\alpha} L^{2}\left(S^{\alpha i}\right)=\left\{v \in L^{2}\left(S^{\alpha i}\right): \Lambda_{\beta}^{\alpha}(g) v=v\right.$ for all $\left.g \in G_{\beta}(S-T)\right\}$. Therefore $P_{T}^{\alpha} \in \Lambda_{\beta}^{\alpha}\left(G_{\beta}\right)^{\prime \prime}$ by Lemma 3 .

LEMMA 5. Let $|S| \geqq \alpha>0$.

1. Let $T \subseteq S$ and $|T|=\alpha$. Then $\Phi \mid L^{2}\left(T^{\alpha b}\right) \cong$ the right regular representation of $G(\alpha)$.

2. $\Phi$ is quasi-equivalent to the right regular representation of $G(\alpha)$. If $\alpha \geqq \aleph_{0}$, then $\Phi$ is a type $\mathrm{II}_{1}$ factor representation of $G(\alpha)$.

3. Let $\beta$ be an infinite cardinal number. Assume $\beta>\alpha$. Then $\Lambda_{\beta}^{\alpha}\left(G_{\beta}\right)^{\prime}=\Phi(G(\alpha))^{\prime \prime}$.

4. Let $\beta$ be an infinite cardinal number and assume $\beta>\alpha$. Then $\Lambda_{\beta}^{\alpha}\left(G_{\beta}(T)\right) \mid L^{2}\left(T^{\alpha b}\right)$ is equivalent to the left regular representation of $G_{\beta}(T)$ if $T \subseteq S$ and $|T|=\alpha$.

5. Let $\beta$ be an infinite cardinal number and assume $\beta \leqq \alpha$. Then $\Lambda_{\beta}^{\alpha}\left(G_{\beta}(T)\right) \mid L^{2}\left(T^{\alpha b}\right)$ is quasi-equivalent to the left regular representation of $G_{\beta}(T)$ if $T \subseteq S$ and $|T|=\alpha$.

Proof. 1. Note that $L^{2}\left(T^{\alpha b}\right)$ is invariant under $\Phi(G(\alpha))$.

Let $f \in T^{\alpha b}$. Let an operator $W$ from $L^{2}\left(T^{\alpha b}\right)$ to $L^{2}(G(\alpha))$ be defined by $W v(p)$ $=v(f p)$, for $v \in L^{2}\left(T^{\alpha b}\right)$ and $p \in G(\alpha)$.W is unitary and implements an equivalence between $\Phi \mid L^{2}\left(T^{\alpha b}\right)$ and the right regular representation of $G(\alpha)$.

2. The first statement is immediate. The second follows from [4, pp. 483-486].

3. Assume $\beta>\alpha$. Let $T \subseteq S$ and assume $|T|=\alpha$. Let $Y \in \Lambda_{\beta}^{\alpha}\left(G_{\beta}\right)^{\prime}$. By Lemma 4 and Lemma 2, to show that $Y \in \Phi(G(\alpha))^{\prime \prime}$, it suffices to show that $Y \mid L^{2}\left(T^{\alpha b}\right)$ $\in \Phi(G(\alpha))^{\prime \prime} \mid L^{2}\left(T^{\alpha b}\right)$.

Let $W$ and $f$ be defined as in the proof of part 1 of this lemma. If $p \in G_{\beta}(T)$, then $W \Lambda_{\beta}^{\alpha}(p) W^{-1}$ is the image under the left regular representation of $G(\alpha)$ of the permutation $f^{-1} p f$ of $\alpha$. Because $\beta>\alpha$, every permutation of $\alpha$ is of the form $f^{-1} p f$ for some $p \in G_{\beta}(T)$. Therefore $W \Lambda_{\beta}^{\alpha}\left(G_{\beta}(T)\right) W^{-1}$ generates the same von Neumann algebra as the left regular representation of $G(\alpha)$. However, the ring generated by 
the left regular representation of $G(\alpha)$ is the commutant of the ring generated by the right regular representation of $G(\alpha)$.

4. Let $T \subseteq S$ and $|T|=\alpha$. Clearly $L^{2}\left(T^{\alpha b}\right)$ is invariant under $\Lambda_{\beta}^{\alpha}\left(G_{\beta}(T)\right)$. Let $f \in T^{\alpha b}$. Let an operator $W$ be defined from $L^{2}\left(T^{\alpha b}\right)$ to $L^{2}\left(G_{\beta}(T)\right)$ by $W v(p)=v(p f)$, for $v \in L^{2}\left(T^{\alpha b}\right)$ and $p \in G_{\beta}(T)$. W is unitary and implements an equivalence between $\Lambda_{\beta}^{\alpha}\left(G_{\beta}(T)\right) \mid L^{2}\left(T^{\alpha b}\right)$ and the left regular representation of $G_{\beta}(T)$.

5. Assume $\alpha \geqq \beta \geqq \aleph_{0}$. Let $T \subseteq S$ and $|T|=\alpha$. Let $W$ be defined as in the proof of part 4 of this lemma. Let $\Gamma_{1}$ be the restriction to $G_{\beta}(T)$ of the left regular representation of $G(T)$. $W$ implements an equivalence between $\Lambda_{\beta}^{\alpha}\left(G_{\beta}(T)\right) \mid L^{2}\left(T^{\alpha b}\right)$ and $\Gamma_{1}$. Let $\Gamma_{2}$ be the right regular representation of $G(T)$.

Let $R C$ be the set of right cosets of $G_{\beta}(T)$ in $G(T)$. Let $C_{1}$ be the right coset which contains the identity. Pick an element $p_{C}$ from each member of $R C$. Then $\Gamma_{1}=\bigoplus_{C \in R C} \Gamma_{1}\left|L^{2}(C)=\bigoplus_{C \in R C} \Gamma_{2}\left(p_{C}^{-1}\right) \Gamma \Gamma_{2}\left(p_{C}\right)\right| L^{2}(C) \sim \Gamma_{1} \mid L^{2}\left(C_{1}\right)=$ the left regular representation of $G_{\beta}(T)$.

Notation. Let $\alpha$ and $\beta$ be cardinal numbers such that $\alpha \geqq 0$ and $\beta \geqq \aleph_{0}$. Let $S^{\# \alpha i}=\left\{f \in S^{|S| i}:|S-f(S)|=\alpha\right\}$. Note that $L^{2}\left(S^{\# \alpha i}\right)$ is a subspace of $L^{2}\left(S^{|S| i}\right)$ and is invariant under $\Lambda_{\beta}^{|S|}\left(G_{\beta}\right)$. If $g \in G_{\beta}$, let $\Lambda_{\beta}^{\# \alpha}(g)$ be the restriction of $\Lambda_{\beta}^{|S|}(g)$ to $L^{2}\left(S^{\# \alpha i}\right)$.

Note that if $T \subseteq S,|T|=|S|$, and $|S-T|=\alpha$, then $T^{|S| b} \subseteq S^{\# \alpha i}$.

In the following theorem, we will express the representations $\Lambda_{\beta}^{\alpha}$ of $G_{\beta}$ as the direct sum of factor representations. The reader is referred to [8, Chapters III and IV] for a discussion of maximal symmetry types and the properties of the regular representation of the symmetric group on $\alpha$ symbols, where $\alpha$ is a positive integer.

THEOREM 2. Let $\alpha$ and $\beta$ be cardinal numbers, where $\alpha>0$ and $\beta \geqq \aleph_{0}$.

1. Assume $\boldsymbol{\aleph}_{0}>\alpha$. Then

a. $\Lambda_{\beta}^{\alpha}\left(G_{\beta}\right)^{\prime \prime}$ is the same von Neumann algebra for all infinite cardinal numbers $\beta$.

b. $\Lambda_{\beta}^{\alpha}$ is the direct sum of irreducible representations of $G_{\beta}$. The irreducible subrepresentations of $\Lambda_{\beta}^{\alpha}$ are in a canonical one-to-one correspondence with the irreducible subrepresentations of the left regular representation of the full symmetric group on a symbols. This one-to-one correspondence and its inverse both preserve equivalence.

2. Assume $|S|>\alpha \geqq \boldsymbol{\aleph}_{0}$.

a. If $\beta>\alpha$, then $\Lambda_{\beta}^{\alpha}$ is a type $\mathrm{II}_{\infty}$ factor representation of $G_{\beta}$.

b. If $\beta \leqq \alpha$, then $\Lambda_{\beta}^{\alpha}$ is the direct sum of infinitely many disjoint type $\mathrm{II}_{\infty}$ factor representations.

3. Assume $\alpha=|S|$.

a. $\Lambda_{\beta}^{\alpha}=\bigoplus_{0 \leqq \gamma \leqq|S|} \Lambda_{\beta}^{\# v}$.

b. If $\beta>\gamma \geqq \aleph_{0}$, then $\Lambda_{\beta}^{\# \gamma}$ is a type $\mathrm{II}_{\infty}$ factor representation.

c. If $|S| \geqq \gamma \geqq \beta$, then $\Lambda_{\beta}^{\# \gamma}$ is the direct sum of infinitely many disjoint type $\mathrm{II}_{\infty}$ factor representations.

d. If $\aleph_{0}>\gamma \geqq 0$, then $\Lambda_{\beta}^{\# \gamma}$ is quasi-equivalent to the left regular representation of $G_{\beta}$. 
4. a. If $0<\alpha, \alpha^{\prime} \leqq|S|$, then $\Lambda_{\beta}^{\alpha}$ o $\Lambda_{\beta}^{\alpha^{\prime}}$ if $\alpha \neq \alpha^{\prime}$.

b. If $0 \leqq \gamma \leqq|S|$ and $\aleph_{0} \leqq \gamma^{\prime} \leqq|S|$ then $\Lambda_{\beta}^{\# \gamma}$ o $\Lambda_{\beta}^{\# \gamma^{\prime}}$.

c. If $0 \leqq \gamma, \gamma^{\prime} \leqq \aleph_{0}$ then $\Lambda_{\beta}^{\# \gamma} \sim \Lambda_{\beta}^{\# \gamma^{\prime}}$.

Proof. 1a. By Lemma 5, part 3, $\Lambda_{\beta}^{\alpha}\left(G_{\beta}\right)^{\prime \prime}=\Phi(G(\alpha))^{\prime}$.

1b. By Lemma 5, part 3, $\Lambda_{\beta}^{\alpha}\left(G_{\beta}\right)^{\prime}=\Phi(G(\alpha))^{\prime \prime}$. By Lemma 5, part 2, $\Phi$ is quasiequivalent to the right regular representation of $G(\alpha)$. Since $\alpha$ is finite, $G(\alpha)$ is a finite group and $\Phi(G(\alpha))^{n}$ is generated by its minimal projections. Consequently, $\Lambda_{\beta}^{\alpha}$ is the direct sum of certain of its irreducible subrepresentations.

Let $T \subseteq S$ be chosen so that $|T|=\alpha$. Then $\Lambda_{\beta}^{\alpha}(G(T)) \mid L^{2}\left(T^{\alpha b}\right)$ is equivalent to the left regular representation of $G(T)$ by Lemma 5, part 4. Let $P$ vary over the set of minimal projections in $\Phi(G(\alpha))^{\prime \prime}$; then $\Lambda_{\beta}^{\alpha}\left|P L^{2}\left(S^{\alpha t}\right) \leftrightarrow \Lambda_{\beta}^{\alpha}(G(T))\right| P L^{2}\left(T^{\alpha b}\right)$ is a oneto-one correspondence between the irreducible subrepresentations of $\Lambda_{\beta}^{\alpha}$ and the irreducible subrepresentations of the left regular representation of the symmetric group on $\alpha$ symbols.

2a. By Lemma 5, part 3, $\Lambda_{\beta}^{\alpha}\left(G_{\beta}\right)^{\prime}=\Phi(G(\alpha))^{\prime \prime}$. By Lemma 5, part 2, $\left.\Phi(G \alpha)\right)^{n}$ is a type $\mathrm{II}_{1}$ factor. Therefore $\Lambda_{\beta}^{\alpha}$ is a type II factor representation of $G_{\beta}$.

Let $T \subseteq S$ and $|T|=\alpha$. By Lemma $4, P_{T}^{\alpha} \in \Lambda_{\beta}^{\alpha}\left(G_{\beta}\right)^{n}$. For each $Z \subseteq S$ such that $|Z|=\alpha$, pick $g_{Z} \in G_{\beta}$ such that $g_{z}(T)=Z$. Then $P_{Z}^{\alpha}=\Lambda_{\beta}^{\alpha}\left(g_{z}\right) P_{T}^{\alpha} \Lambda_{\beta}^{\alpha}\left(g_{z}^{-1}\right) . P_{Z}^{\alpha} P_{T}^{\alpha}=0$ if $Z \neq T$. Therefore $\Lambda_{\beta}^{\alpha}\left(G_{\beta}\right)^{\prime \prime}$ contains infinitely many mutually perpendicular nonzero equivalent projections and consequently cannot be of finite type.

2b. Let $\mathscr{S}=\{T \subseteq S:|T|=\alpha\}$. Define an equivalence relation \pm on $\mathscr{S}$ by $T \perp Q$ iff $|(T-Q) \cup(Q-T)|<\beta$ iff $\exists g \in G_{\beta}$ such that $g(T)=Q$. Let $\left\{C_{\delta}: \delta \in \Delta\right\}$, where $\Delta$ is an index set, be the set of equivalence classes of $\perp$ in $S . \Delta$ is an infinite set because $\beta \leqq \alpha$. If $\delta \in \Delta$, let $H_{\delta}=\bigoplus_{T \in \delta} L^{2}\left(T^{\alpha b}\right)$. Clearly $L^{2}\left(S^{\alpha i}\right)=\bigoplus_{\delta \in \Delta} H_{\delta} . H_{\delta}$ is invariant under $\Lambda_{\beta}^{\alpha}\left(G_{\beta}\right)$. Therefore $\Lambda_{\beta}^{\alpha}=\bigoplus_{\delta \in \Delta} \Lambda_{\beta}^{\alpha} \mid H_{\delta}$.

By Lemma 4 and Lemma $2, \Lambda_{\beta}^{\alpha}\left(G_{\beta}\right)^{\prime} \mid H_{\delta}$ is algebraically *-isomorphic to $\Lambda_{\beta}^{\alpha}\left(G_{\beta}(T)\right)^{\prime} \mid L^{2}\left(T^{\alpha b}\right)$. By Lemma 5, part $5, \Lambda_{\beta}^{\alpha} \mid H_{\delta}$ is a type II factor representation. The proof that $\Lambda_{\beta}^{\alpha} \mid H_{\delta}$ is type $\mathrm{II}_{\infty}$ is similar to the last part of the proof of part $2 \mathrm{a}$ of this theorem and is left to the reader.

Let $T \in \mathscr{S}$ and $v \in L^{2}\left(T^{\alpha b}\right)$. Then $\Lambda_{\beta}^{\alpha}(g) v=v$ if $g \in G_{\beta}(S-T)$. Let $Y \in \mathscr{S}$. Then $\Lambda_{\beta}^{\alpha}(p) v=v$ for all $p \in G_{\beta}(S-Y)$ iff $Y \supseteq T$. If $\delta \in \Delta, T_{1} \in \delta, T_{2} \in \delta, T_{3} \in \mathscr{S}$, and $T_{1} \supseteq T_{3} \supseteq T_{2}$, then clearly $T_{3} \in \delta$. It follows from Lemma 1 that $\Lambda_{\beta}^{\alpha}\left|H_{\delta} \delta \Lambda_{\beta}^{\alpha}\right| H_{\delta^{\prime}}$ if $\delta, \delta^{\prime} \in \Delta$ and $\delta \neq \delta^{\prime}$.

3. Part $a$ is trivial. The proofs of parts $b$ and $c$ are similar to the proofs of $2 b$ and $2 \mathrm{c}$ and are left to the reader.

3d. Let $\gamma=0$. By Lemma 5, parts 4 and $5, U^{\# 0}$ is quasi-equivalent to the left regular representation of $G_{\beta}$ and so is a type $\mathrm{II}_{1}$ factor representation.

Assume $0<\gamma<\boldsymbol{\aleph}_{0}$. Let $\theta$ be any mapping from $S^{\# v i}$ to $S^{\# 0 i}$ such that, if $f \in S^{\# r i}$, then $(\theta f)(\delta)=f(\delta)$ if $\delta$ is an ordinal and $|S|>\delta \geqq \boldsymbol{\aleph}_{0}$, and $\theta f(\delta)=f(\delta+\gamma)$ if $\delta$ is an ordinal and $\delta<\boldsymbol{\aleph}_{0} . \theta$ is one-to-one. Let $W$ be the unique operator from $L^{2}\left(S^{\# \gamma i}\right)$ into $L^{2}\left(S^{\# 0 t}\right)$ such that $W \chi(\{f\})=\gamma !^{-1 / 2} \sum_{g \in G(\gamma)} \Phi(g) \chi(\{\theta(f)\})$, for $f \in S^{\# \nu i}$, where $\chi$ denotes characteristic function. 
$W$ is an isometry from $L^{2}\left(S^{\# \gamma^{i}}\right)$ to $L^{2}\left(S^{\# 0 i}\right)$. Therefore, $W$ implements an equivalence between $\Lambda_{\beta}^{\# \gamma}$ and a subrepresentation of $\Lambda_{\beta}^{\# 0}$. Since $\Lambda_{\beta}^{\# 0}$ is a factor representation, it is quasi-equivalent to any of its subrepresentations.

4a. Assume $Q \subseteq S$ and $|Q|<\alpha$. Let $v \in L^{2}\left(S^{\alpha i}\right), v \neq 0$. Then $\exists g \in G_{\beta}(S-Q)$ such that $\Lambda_{\beta}^{\alpha}(g) v \neq v$.

$L^{2}\left(S^{\alpha i}\right)=\oplus P_{T}^{\alpha} L^{2}\left(S^{\alpha i}\right)$, where the direct sum is taken over all subsets $T$ of $S$ such that $|T|=\alpha$. If $\Gamma$ is any subrepresentation of $\Lambda_{\beta}^{\alpha}$, then there is a subset $Z$ of $S$ with $|Z|=\alpha$ and a nonzero vector $w$ in the subspace on which $\Gamma$ acts such that $P_{Z}^{\alpha} w=w$. Then $\Gamma(g) w=w$ for all $w \in G_{\beta}(S-Z)$. The conclusion is an immediate consequence of Lemma 1.

4b. The proof of $4 b$ is similar to the proof of $4 a$ and is left to the reader.

4c. This is trivial.

IV. The continuous unitary representations of the infinite symmetric groups. Give $G_{\beta}$ the topology of pointwise convergence on $S$. If $s \in S$, then $\left\{g \in G_{\beta}: g(s)=s\right\}$ is a subbasic open neighborhood of the identity. $G_{\beta}$ is a topological group in this topology but is not locally compact. $G_{\aleph_{0}}$ is dense in $G_{\beta} . G_{\beta}$ is complete as a uniform space iff $G_{\beta}$ is the group of all permutations of $S$. A representation of $G_{\beta}$ is continuous if it is continuous with respect to this topology on $G_{\beta}$ and the weak operator topology on the unitary group of Hilbert space.

We define the trivial representation of any group as the one-dimensional representation which maps every element of the group to the identity operator. $\Lambda_{\beta}^{0}$ will denote the trivial representation of $G_{\beta}$.

THEOREM 3. Let $\beta$ be an infinite cardinal number. Let $\Gamma$ be a continuous irreducible representation of $G_{\beta}$ on the Hilbert space $H$. Then there is a nonnegative integer $n$ such that

1. $\Gamma$ is equivalent to an irreducible subrepresentation of $\Lambda_{\beta}^{n}$.

2. There is a subset $Z$ of $S$ such that $|Z|=n$ and the restriction of $\Gamma$ to $G_{\beta}(S-Z)$ contains the trivial representation of $G_{\beta}(S-Z)$.

3. If $Z$ is any subset of $S$ such that $|Z| \geqq n$, then the restriction of $\Gamma$ to $G_{\beta}(S-Z)$ contains the trivial representation of $G_{\beta}(S-Z)$.

Conversely, if the irreducible representation $\Gamma$ of $G_{\beta}$ satisfies condition 1 , condition 2 , or condition 3 , then $\Gamma$ is continuous.

THEOREM 4. Let $\Gamma$ be a continuous representation of $G_{\beta}$. Then $\Gamma$ is the direct sum of irreducible representations of $G_{\beta}$.

Two lemmas are needed to prove these theorems. If $n$ is a positive integer, $\mathscr{S}_{n}$ will denote $\{Q \subseteq S:|Q|=n\}$.

Lemma 6. Let $H_{i}, i=1,2$, be a Hilbert space and let $\Gamma_{i}$ be a representation of $G_{\beta}$ on $H_{i}$. Assume 
1. There is a positive integer $n$ such that, for $i=1,2, H_{i}=\bigoplus_{Q \in \mathscr{P}_{n}} H_{i Q}$, where $H_{i Q}=\left\{v \in H_{i}: \Gamma_{i}(g) v=v\right.$ for all $\left.g \in G_{\beta}(S-Q)\right\}$.

2. There is a subset $T$ of $S$ such that $|T|=n$ and $\Gamma_{1}\left(G_{\beta}(T)\right)\left|H_{1 T} \cong \Gamma_{2}\left(G_{\beta}(T)\right)\right| H_{2 T}$. Then $\Gamma_{1} \cong \Gamma_{2}$.

Proof. In Lemma 2, let $G=G_{\beta}, H=H_{1} \oplus H_{2}, \Gamma=\Gamma_{1} \oplus \Gamma_{2}$, and $J=H_{1 T} \oplus H_{2 T}$. Then $P$ is projection onto $H_{1 T} \oplus H_{2 T}$, and, by assumption $1, G_{J}=\left\{g \in G_{\beta}: g(T)=T\right\}$.

Assumption a of Lemma 2 follows from $|T|=n<\boldsymbol{\aleph}_{0} \leqq \beta$ and assumption 1 of this lemma. Assumption b follows immediately from assumption 1 of this lemma; c follows from Lemma 3.

Let $W$ be a unitary operator from $H_{1 T}$ onto $H_{2 T}$ which implements the equivalence in assumption 2. Let $U$ be the unique operator on $H_{1 T} \oplus H_{2 T}$ such that $U x=W x$ if $x \in H_{1 T}$ and $U x=0$ if $x \in H_{2 T}$. Then $U \in \Gamma\left(G_{J}\right)^{\prime} \mid J$. By Lemma 2, there is an operator $U_{0} \in\left(\left(\Gamma_{1} \oplus \Gamma_{2}\right)\left(G_{\beta}\right)\right)^{\prime}$ such that $U_{0} \mid J=U$. Let $W_{0}=U_{0} \mid H_{1}$. $W_{0}$ implements an equivalence between $\Gamma_{1}$ and $\Gamma_{2}$.

Lemma 7. Let $\Gamma$ be a representation of $G_{\beta}$ on the Hilbert space $H$. Let $n$ be a positive integer. Assume

a. The subspace spanned by $\bigcup_{Q \in \mathscr{S}_{n}} H_{Q}$ is dense in $H$, where

$$
H_{Q}=\left\{x \in H: \Gamma(g) x=x \text { for all } g \in G_{\beta}(S-Q)\right\}
$$

b. $H_{Z}=0$ if $Z \subseteq S$ and $|Z|<n$.

Then $H=\oplus_{Q \in \mathscr{S}_{n}} H_{Q}$.

Proof. Let $T_{1}, T_{2} \subseteq S,\left|T_{1}\right|=\left|T_{2}\right|=n$, and $T_{1} \neq T_{2}$. Assume $H_{T_{1}}$ is not orthogonal to $H_{T_{2}}$. Let $R=T_{1} \cap T_{2}$. Note that $|R|<n$. Pick a sequence $T_{3}, T_{4}, \ldots$ of members of $\mathscr{S}_{n}$ such that $T_{j} \cap T_{k}=R$ for $j \neq k, j, k=1,2,3,4, \ldots$ Let $P_{j}$ be projection onto $H_{T_{j}}$. For $j \geqq 3$, pick $g_{j} \in G_{\beta}$ such that $g_{j}\left(T_{2}\right)=T_{j}$ and $g_{j}$ leaves $T_{1}$ pointwise fixed.

Because $H_{T_{1}}$ and $H_{T_{2}}$ are not orthogonal, $\exists w \in H_{T_{1}}$ such that $P_{2} w \neq 0$. Let $x=P_{2} w$. The unitary operator $\Gamma\left(g_{j}\right)$ maps $H_{T_{2}}$ onto $H_{T_{1}}$ for $j \geqq 3$, and consequently maps $\left(I-P_{2}\right) H$ onto $\left(I-P_{j}\right) H$ for $j \geqq 3$.

Let $j \geqq 3$. Then $P_{j} w=P_{j} \Gamma\left(g_{j}\right) w=P_{j} \Gamma\left(g_{j}\right) x+P_{j} \Gamma\left(g_{j}\right)(w-x)=\Gamma\left(g_{j}\right) x+0=\Gamma\left(g_{j}\right) x$. If $P_{j} w=P_{k} w$ for some $j, k$ such that $j \neq k$, then $P_{j} w \in H_{\left(T_{j} \cap T_{k}\right)}=H_{R}$. This would contradict assumption b. Since $\left\|P_{j} w\right\|=\|x\| \neq 0$, for $j \geqq 3,\left\{P_{j} w \mid j \geqq 3\right\}$ has a weak limit point $y . \quad y \neq 0$ because $|(y, w)| \geqq \liminf _{j}\left|\left(P_{j} w, w\right)\right|=\lim \inf _{j}\left\|P_{j} w\right\|^{2}$ $=\lim \inf _{j}\|x\|^{2}=\|x\|^{2} \neq 0$.

Let $g \in G_{\aleph_{0}}(S-R)$. Since $g$ is finitely supported and $T_{j} \cap T_{k}=R$ if $j \neq k, g$ leaves $T_{j}$ pointwise fixed for $j$ sufficiently large. Therefore $\Gamma(g) P_{j}=P_{j}$ for $j$ sufficiently large. This implies that $\Gamma(g) y=y$.

By assumption a, there are scalars $a_{Q}$ and vectors $x_{Q}$ such that $x_{Q} \in H_{Q},\left\|x_{Q}\right\|=1$, and $y=\sum_{Q \in \mathscr{S}_{n}} a_{Q} x_{Q}$. Let $p \in G_{\beta}(S-R)$ and $c>0$ be given. Let $\mathscr{Y}$ be a finite subset 
of $\mathscr{S}_{n}$ such that $\left\|\sum_{Q \in \in} \mathscr{S}_{n}-\mathscr{Y} a_{Q} x_{Q}\right\|<c / 2$. Pick $h \in G_{\aleph_{0}}(S-R)$ such that $g h^{-1}$ leaves $Q$ pointwise fixed if $Q \in \mathscr{Y}$. Then

$$
\begin{aligned}
\|\Gamma(g) y-y\| & =\left\|\Gamma(g) \Gamma\left(h^{-1}\right) y-y\right\|=\left\|\Gamma\left(g h^{-1}\right) y-y\right\| \\
& =\left\|\left(\Gamma\left(g h^{-1}\right)-I\right) \sum_{Q \in \mathscr{S}_{n}} a_{Q} x_{Q}\right\| \\
& \leqq\left\|\left(\Gamma\left(g h^{-1}\right)-I\right) \sum_{Q \in \mathscr{Y}} a_{Q} x_{Q}\right\|+\left\|\left(\Gamma\left(g h^{-1}\right)-I\right) \sum_{Q \in \mathscr{P}_{n}-\mathscr{g}} a_{Q} x_{Q}\right\| \\
& <0+2(c / 2)=c .
\end{aligned}
$$

Since $c$ is arbitrary, $\Gamma(g) y=y$ for all $g \in G_{\beta}(S-R)$. This contradicts assumption b.

Proof of Theorem 3. Assume $\Gamma$ is a continuous irreducible representation of $G_{\beta}$ on the Hilbert space $H$. Let $\mathscr{Z}$ be the directed set of all finite subsets $S$ with set inclusion as the partial order relation. Let $v \in H,\|v\|=1$. Assume that for each $T \in \mathscr{Z}$ there is a permutation $g_{T} \in G_{\beta}(S-T)$ such that $\operatorname{re}\left(\Gamma\left(g_{T}\right) v, v\right) \leqq \frac{1}{2}$. Then $\lim \sup _{T \in \mathscr{Z}} \operatorname{re}\left(\Gamma\left(g_{T}\right) v, v\right) \leqq \frac{1}{2}$. However $\lim _{T \in \mathscr{Z}} g_{T}=e$, where $e$ is the group identity. Therefore, $\lim _{T \in \mathscr{Z}}\left(\Gamma\left(g_{T}\right) v, v\right)=(\Gamma(e) v, v)=1$, yielding a contradiction. Therefore, there is a finite subset $Z$ of $S$ such that $\operatorname{re}(\Gamma(g) v, v) \geqq \frac{1}{2}$ if $g \in G_{\beta}(S-Z)$.

Let $\mathscr{Y}$ be the directed set of all finite subsets of $S-Z$ with set inclusion as the partial order relation. If $T \in \mathscr{Y}$, let $P_{T}=|T| !^{-1} \sum_{g \in G(T)} \Gamma(g) . P_{T}$ is a projection and $P_{T} \in \Gamma\left(G_{\beta}\right)^{\prime \prime} . P_{T_{1}} P_{T}=P_{T_{1}}$ if $T_{1} \in \mathscr{Y}$ and $T_{1} \supseteq T$. $\Gamma(g) P_{T}=P_{T}$ if $g \in G(T)$.

Let $J=\left(\bigcup_{Q \in \mathscr{Y}}\left(I-P_{Q}\right) H\right)^{\perp}$ and let $P$ be projection onto $J$. Let $x \in J$. Then $\left(I-P_{Q}\right) x=0$ for all $Q \in \mathscr{Y}$. Let $y \in J^{\perp}$ and $c>0$ be given. There exist $Q_{1}, Q_{2}, \ldots$, $Q_{m} \in \mathscr{Y}$, where $m$ is some positive integer, such that $\left\|P^{\prime} y-y\right\|<c$, where $P^{\prime}$ is projection onto the subspace spanned by $\bigcup_{j=1}^{m}\left(I-P_{Q_{j}}\right) H$. Let $Q^{\prime}=\bigcup_{j=1}^{m} Q_{j}$. Then $\left\|\left(I-P_{Q^{\prime}}\right) y-y\right\|<c$. Therefore $\left\|P_{Q^{\prime}} y\right\|<c$.

Let $Q \in \mathscr{Y}$ and assume $Q \supseteq Q^{\prime}$. Then $P_{Q} y=P_{Q} P_{Q^{\prime}} y$ and $\left\|P_{Q} y\right\|<c$. Consequently, $\lim _{Q \in \mathscr{Y}} P_{Q}(y)=0$ and therefore $P=\lim _{Q \in \mathscr{Y}} P_{Q}$.

re $(P y, y) \geqq \inf _{Q \in \mathscr{Y}}\left(P_{Q} y, y\right) \geqq \frac{1}{2}$. Therefore $P y \neq 0$. Let $v=P y, T \in \mathscr{Y}$, and $g \in G(T)$. Then $\Gamma(g) v=\Gamma(g) P y=\Gamma(g) \lim _{Q \in \mathcal{G}}\left(P_{Q} y\right)=\lim _{Q \in \mathcal{G}}\left(\Gamma(g) P_{Q} y\right)=\lim _{Q \in \in}\left(P_{Q} y\right)=P y$ $=v$, since $\Gamma(g) P_{Q}=P_{Q}$ if $Q \supseteq T$. By continuity, $\Gamma(p) v=v$ if $p \in G_{\beta}(S-Z)$.

Without loss of generality, we can assume that $H_{Q}=0$ if $Q \subseteq S$ and $|Q|<|Z|$, where $H_{Q}=\left\{x \in H: \Gamma(g) x=x\right.$ for $\left.g \in G_{\beta}(S-Q)\right\}$. Let $|Z|=n$. Conclusion 2 of the theorem has been demonstrated; conclusion 3 is an immediate consequence.

The closed subspace spanned by $\bigcup_{Q \in \mathscr{S}_{n}} H_{Q}$ is invariant under $\Gamma\left(G_{\beta}\right)$. Since $\Gamma$ is irreducible, this subspace must be equal to $H$. By Lemma $7, H=\bigoplus_{Q \in \mathscr{S}_{n}} H_{Q}$.

By Lemma 3 and Lemma $2, \Gamma(G)^{\prime} \cong \Gamma(G(Z))^{\prime} \mid H_{z}$. Since $\Gamma$ is irreducible, $\Gamma(G)^{\prime}$ is the set of scalar multiples of the identity operator and $\Gamma(G(Z)) \mid H_{Z}$ is irreducible.

If $Z=\varnothing$ then $H_{Z}=H$ and $\Gamma \cong \Lambda_{\beta}^{0}$. If $Z \neq \varnothing$, let $\psi$ be the subrepresentation of $\Lambda_{\beta}^{n}$ which corresponds to $\Gamma(G(Z)) \mid H_{Z}$ as defined in Theorem 2 part $1 \mathrm{~b}$. Let $J$ be the Hilbert space on which $\psi$ acts.

In Lemma 6, let $H_{1}=J, H_{2}=H, \Gamma_{1}=\psi, \Gamma_{2}=\Gamma, n=n$, and $T=Z$. The assumptions of Lemma 6 are satisfied, and consequently $\psi \cong \Gamma$. 
We now prove the converse of the theorem when condition 2 is satisfied. The remainder of the proof will then be immediate. Assume that $\Gamma$ is an irreducible representation of $G_{\beta}, Z \in \mathscr{S}_{n}$, and $\Gamma\left(G_{\beta}(S-Z)\right)$ contains the trivial representation of $G_{\beta}(S-Z)$. This implies $H_{Z} \neq 0$. Without loss of generality, we can assume $H_{T}=0$ if $T \subseteq S$ and $|T|<|Z|$. The closed subspace generated by $\bigcup_{T \in \mathscr{S}_{n}} H_{T}$ is $\neq 0$ and is invariant under $\Gamma\left(G_{\beta}\right)$; consequently, this subspace is all of $H$. By Lemma 7, $H=\bigoplus_{T \in \mathscr{S}_{n}} H_{T}$.

Let $x \in H$ and $c>0$ be given. We can find sets $T_{i} \in \mathscr{S}_{n}$ and vectors $x_{i} \in H_{T_{i}}$, $1 \leqq i \leqq m, m$ some positive integer, such that $\left\|x-\sum_{i=1}^{m} x_{i}\right\|<c / 2$. Let $0=\left\{g \in G_{\beta}\right.$ : $g(s)=s$ for all $\left.s \in \bigcup_{i=1}^{m} T_{i}\right\} .0$ is an open neighborhood of the identity. If $g \in 0$, then

$$
\begin{aligned}
\|\Gamma(g) x-\Gamma(e) x\| & =\|(\Gamma(g)-I) x\|=\left\|(\Gamma(g)-I)\left(x-\sum_{i=1}^{m} x_{i}\right)+(\Gamma(g)-I) \sum_{i=1}^{m} x_{i}\right\| \\
& \leqq\|\Gamma(g)-I\|\left\|x-\sum_{i=1}^{m} x_{i}\right\|+0<2 \cdot c / 2=c .
\end{aligned}
$$

Therefore $\Gamma$ is continuous.

Proof of Theorem 4. It suffices to show that $\Gamma$ contains an irreducible subrepresentation. An application of Zorn's lemma will then complete the proof. Assume $\Gamma$ acts on the Hilbert space $H$.

The proof of conclusion 2 of Theorem 3 never used the hypothesis of irreducibility. Consequently, we can assume there is a finite subset $Z$ of $S$ such that $H_{Z} \neq 0$ and $H_{Q}=0$ if $Q \subseteq S$ and $|Q|<|Z| . G(Z)$ is a finite group, and consequently $\Gamma(G(Z)) \mid H_{Z}$ is the direct sum of irreducible representations of $G(Z)$. Let $\Gamma_{0}$ be an irreducible subrepresentation of $\Gamma(G(Z)) \mid H_{z}$. Assume $\Gamma_{0}$ acts on $H_{0 z}$. Let $H_{0}$ be the closed subspace of $H$ generated by $\Gamma(G) H_{0 z}$. By Lemma 3 and Lemma 2, $\Gamma \mid H_{0}$ is irreducible.

\section{BIBLIOGRAPHY}

1. J. Dixmier, Les algèbres d'opérateurs dans l'espace Hilbertien, 2nd ed., Gauthier-Villars, Paris, 1969.

2. - Les $C^{*}$-algèbres et leurs représentations, Cahiers Scientifique, Fasc. 29, GauthierVillars, Paris, 1964. MR 30 \#1404.

3. K. Gödel, The consistency of the continuum hypothesis, Ann. of Math. Studies, no. 3, Princeton Univ. Press, Princeton, N. J., 1940. MR 2, 66.

4. M. A. Naĭmark, Normed rings, GITTL, Moscow, 1956; English transl., Noordhoff, Groningen, 1959. MR 19, 870; MR 22 \#1824.

5. G. B. Robinson, Representation theory of the symmetric group, Math. Expositions, no. 12, University of Toronto Press, Toronto, 1961. MR 23 \#A3182.

6. I. E. Segal, The structure of a class of representations of the unitary group on a Hilbert space, Proc. Amer. Math. Soc. 8 (1957), 197-203. MR 18, 812.

7. E. Thoma, Die unzerlegbaren, positiv-definiten Klassenfunctionen der abzählbar unendlichen symmetrischen Gruppe, Math. Z. 85 (1964), 40-61. MR 30 \#3382.

8. H. Weyl, The classical groups. Their invariants and representations, 2nd ed., Princeton Univ. Press, Princeton, N. J., 1946. MR 1, 42.

Department of Mathematics, University of South Florida, Tampa, Florida 33620 